\title{
Practice-Related Improvements in Somatosensory Interval Discrimination Are Temporally Specific But Generalize across Skin Location, Hemisphere, and Modality
}

\author{
Srikantan S. Nagarajan, David T. Blake, Beverly A. Wright, Nancy Byl, and Michael M. Merzenich \\ Coleman Laboratory, Keck Center for Integrative Neuroscience, University of California, San Francisco, \\ California 94143-0732
}

\begin{abstract}
This paper concerns the characterization of performance and perceptual learning of somatosensory interval discrimination. The purposes of this study were to define (1) the performance characteristics for interval discrimination in the somatosensory system by naive adult humans, (2) the normal capacities for improvement in somatosensory interval discrimination, and (3) the extent of generalization of interval discrimination learning. In a two-alternative forced choice procedure, subjects were presented with two pairs of vibratory pulses. One pair was separated in time by a fixed base interval; a second pair was separated by a target interval that was always longer than the base interval. Subjects indicated which pair was separated by the target interval. The length of the target interval was varied adaptively to determine discrimination thresholds. After initial determination of naive abilities, subjects were trained for 900
\end{abstract}

trials per day at base intervals of either 75 or $125 \mathrm{msec}$ for 10-15 d. Significant improvements in thresholds resulted from training. Learning at the trained base interval generalized completely across untrained skin locations on the trained hand and to the corresponding untrained skin location in the contralateral hand. The learning partially generalized to untrained base intervals similar to the trained one, but not to more distant base intervals. Learning with somatosensory stimuli generalized to auditory stimuli presented at comparable base intervals. These results demonstrate temporal specificity in somatosensory interval discrimination learning that generalizes across skin location, hemisphere, and modality.

Key words: somatosensory; vibrotactile; temporal processing; perceptual learning; psychophysics; tactile; touch; human; auditory; hearing; interval; time
Durations of stimuli and intervals between stimuli provide important cues for temporal information processing of complex signals in the brain. A classic example of such processing is speech recognition in which temporal cues, such as consonant-vowel transition durations and pauses, provide crucial information to the perceiver (Licklider, 1951; Tallal et al., 1993; Drullman, 1995; Shannon et al., 1995). It is surprising therefore that relatively little is known about the neurobiological bases for the processing of temporal cues in stimuli, especially in the time scale of 50-500 msec, for which it is hypothesized that cortical mechanisms are invoked (Mountcastle, 1993; Ivry, 1996; Gibbon et al., 1997). In addition to being a fundamental issue in neuroscience, determination of the neurobiological basis of temporal information processing has important practical implications. For instance, deficits in temporal processing commonly are associated with languagelearning impairments in children and adults (Tallal, 1990; Wright et al., 1997b) and can be ameliorated by training (Merzenich et al., 1996). Furthermore, knowledge of the temporal information processing abilities in different sensory systems could help to

Received Aug. 1, 1997; revised Nov. 11, 1997; accepted Nov. 24, 1997.

This research was supported by National Institutes of Health Grants NS-10414 and R29-DC02997, Hearing Research Incorporated, McDonnell-Pew Program in Cognitive Neuroscience, and the Coleman Fund. We thank Drs. Dean Buonomano and Peter Bachietti for help with statistical analyses and Dr. Merav Ahissar for comments on an earlier version of this manuscript.

Correspondence should be addressed to Dr. Srikantan Nagarajan, Coleman Laboratory, Keck Center for Integrative Neuroscience, 513 Parnassus Avenue, S877, University of California, San Francisco, CA 94143-0732.

Dr. Wright's present address: Audiology and Hearing Sciences Program, Northwestern University, 2299 North Campus Drive, Evanston, IL 60208-3550.

Copyright (c) 1998 Society for Neuroscience $0270-6474 / 98 / 181559-12 \$ 05.00 / 0$ guide the design of prosthetic devices, using sensory stimulation (Koczmarec et al., 1991; Minagawa et al., 1996).

So that the fundamental principles governing the cortical representation of temporal information can be derived, it is important to characterize temporal information processing abilities in the visual, auditory, somatosensory, and motor systems. However, to date, performance in temporal tasks, such as duration discrimination, interval discrimination, and time estimation, has been examined predominantly with auditory stimuli; there have been fewer studies in the visual, somatosensory, and motor systems (Allan, 1979, 1992; Gibbon and Church, 1990; Ivry, 1996).

There is also a lack of information about the characteristics of learning attributable to practice at temporal tasks. The psychophysics of such perceptual learning can provide crucial insights into the neurobiological basis of temporal information processing. For example, perceptual learning in auditory temporal interval discrimination was reported recently in normal adult humans (Wright et al., 1997a). This learning generalized to the trained interval that was presented two octaves from the trained frequency, but it did not generalize to an untrained interval that was presented at the trained frequency. Thus, the learning in auditory interval discrimination was temporal, but not spatially (spectrally) specific.

If temporal information processing is governed by the same mechanisms across different sensory systems, then naive subjects should show similar discrimination thresholds and similar patterns of learning and generalization with somatosensory and auditory stimuli. Therefore, the goals of the present study were to (1) determine interval discrimination thresholds with somatosensory and auditory stimuli in naive adults, (2) document training- 
induced changes in interval discrimination thresholds with somatosensory stimuli, and (3) establish whether improvements in somatosensory interval discrimination generalize to untrained skin locations, untrained intervals, or an untrained modality (audition). The results show that naive subjects have similar interval discrimination thresholds for somatosensory and auditory stimuli and that the patterns of learning and generalization of somatosensory interval discrimination are parallel to those previously seen for auditory interval discrimination.

\section{MATERIALS AND METHODS}

Subjects. Twenty-two volunteers between the ages of 19 and 51 years served as subjects. Two subjects (S10 and S14) were the first and second authors; the rest were paid for their participation. All of the subjects except the authors had no previous experience with somatosensory psychophysics or interval discrimination tasks.

Stimuli. Stimuli were presented either to the thenar eminence (the region of the palm that lies in a line between the thumb and the wrist) or to the distal tips of the third digit (middle finger) or fourth digit (ring finger). Sinusoidal vibrotactile stimuli were presented with a flat tip aluminum contact with a diameter of $2.5 \mathrm{~mm}$. The contact was centered in a rigidly secured surround made of acrylic, which had a diameter of 7 $\mathrm{mm}$. The contact and the surround were mounted on a lever arm that was counterbalanced to deliver a constant force of $100 \mathrm{gms}$. Displacements of the contacts were generated by custom-built displacement feedback tactile stimulators controlled by LabView software on a Macintosh computer. To ensure that each somatosensory stimulus was clearly detectable, we set peak displacement at $100 \mu \mathrm{m}$ above a static indentation of $\sim 1 \mathrm{~mm}$ after skin contact. Skin contact was determined by using an impedance measurement between the body and the metal probe. Although easily audible sounds were not produced by any of the stimuli used in these experiments, masking noise was presented through circumaural headphones to eliminate possible auditory cues. The masking noise was a low-pass-filtered pink noise set at a comfortable level by the subject.

Two pairs of somatosensory pulses were presented to the subject on each trial. Each brief pulse comprised one sinusoidal cycle and had a maximum displacement of $100 \mu \mathrm{m}$, a frequency of $40 \mathrm{~Hz}$, and a total duration of $25 \mathrm{msec}$, including $5 \mathrm{msec}$ rise/fall ramps. The interval marked by the pulses in a pair was measured from the onset of the first pulse to the onset of the second pulse.

Procedure. One pair of somatosensory pulses was presented in each observation period of a two-alternative forced choice trial (2AFC). In one observation period the two pulses of the pair were separated by a fixed interval referred to as the "base interval." In the other observation period the two pulses of the pair were separated by a target interval that was always longer than the base interval. The target interval was presented randomly either in the first or the second observation period. Subjects signaled which of the two observation periods contained the target interval by pressing a button on an external interface board. A light-emitting diode provided response feedback at the end of each trial. The target interval was the adaptive parameter that was decreased when the subject responded correctly on three consecutive trials and was increased whenever the subject responded incorrectly. The value of the target interval on trials in which the direction of the adaptive parameter changed from increasing to decreasing or vice versa was referred to as a reversal. This "three-down/one-up" rule estimates the $79 \%$ correct point on the psychometric function (Levitt, 1971). The step size was $10 \%$ of the base interval until the third reversal and was $1 \%$ of the base interval thereafter. At the start of each block of trials the target and base intervals were equal, forcing the subject to guess on the first few trials. The first three reversals of each 60 trial block were discarded, and thresholds were estimated by taking the average of the remaining even number of reversals. A threshold estimate was retained from a given block only if there were more than seven total reversals in a block. Thresholds are expressed as Weber fractions, defined as:

$$
\text { Weber fraction }=\frac{\text { Target interval at threshold }- \text { Base interval }}{\text { Base interval }} .
$$

At the first experimental session, to familiarize themselves with the experimental apparatus and the 2AFC experimental paradigm, subjects performed a vibrotactile detection task on the skin location in which they
Table 1. Pre- and post-test base intervals measured in each subject listed by group ${ }^{a}$

\begin{tabular}{|c|c|c|c|c|}
\hline \multirow{2}{*}{$\frac{\text { Subjects }}{\text { Group tr }}$} & \multicolumn{4}{|l|}{ Skin location } \\
\hline & \multicolumn{4}{|c|}{ Group trained at $125 \mathrm{msec}$ on left digit 4} \\
\hline \multirow[t]{3}{*}{ S1-S7 } & $\begin{array}{l}\text { Left digit } 4 \\
75\end{array}$ & Left digit 3 & Right digit 4 & $\begin{array}{l}\text { Auditory } \\
50\end{array}$ \\
\hline & 125 & 125 & 125 & 100 \\
\hline & 225 & & & 200 \\
\hline \multirow[t]{3}{*}{ S8 } & $\begin{array}{l}\text { Left digit } 4 \\
75\end{array}$ & Left digit 3 & Left thenar & \\
\hline & 125 & 125 & 125 & \\
\hline & 225 & & & \\
\hline \multicolumn{5}{|c|}{ Group trained at $125 \mathrm{msec}$ on left thenar eminence } \\
\hline \multirow[t]{3}{*}{ S9-S12 } & $\begin{array}{l}\text { Left thenar } \\
75\end{array}$ & Right thenar & & \\
\hline & 125 & 125 & & \\
\hline & 225 & & & \\
\hline \multicolumn{5}{|c|}{ Group trained at $75 \mathrm{msec}$ on left digit 4} \\
\hline \multirow[t]{2}{*}{ S13-S16 } & $\begin{array}{l}\text { Left digit } 4 \\
75\end{array}$ & Right digit 4 & & \\
\hline & 125 & 125 & & \\
\hline \multicolumn{5}{|c|}{ Control group (no training) } \\
\hline \multirow[t]{3}{*}{ S17-S22 } & $\begin{array}{l}\text { Left digit } 4 \\
75\end{array}$ & Left digit 3 & Right digit 4 & \\
\hline & 125 & 125 & 125 & \\
\hline & 225 & & & \\
\hline
\end{tabular}

${ }^{a}$ Base intervals tested at each skin location are shown. Only subjects who participated in the post-test phase are listed.

were to be trained. This procedure was used to establish normalcy in somesthetic sensation. The main experiment was initiated at the second session and consisted of pretest, training, and post-test phases. Pretest measurements determined the psychophysical thresholds for interval discrimination for naive subjects. For different subjects the pretest, training, and post-test measurements were determined for stimuli delivered to different skin locations, base intervals, and hands. Of the 22 subjects, 16 participated in all three phases, and six participated in the pre- and post-tests with no training. The various conditions tested during the pre- and post-test phases for all of the subjects are listed in Table 1. During the pre- and post-test phases the thresholds for the condition at which the subject was to be trained were always collected first; thresholds at a corresponding skin location in the opposite hand were collected last (with the exception of subject S8, in which thresholds at the left thenar eminence at a base interval of $125 \mathrm{msec}$ were measured last). The rest of the conditions were presented in a pseudo-random order. Thresholds in the pre- and post-test phases were estimated from five or six blocks per condition.

The training phase occurred between the pre- and post-test phases. It consisted of $1 \mathrm{hr}$ of practice per day for $10-16 \mathrm{~d}$ at discriminating longer intervals from a particular base interval. Fifteen to sixteen threshold estimates (900-960 trials) were collected within each training session. The position of the applied stimuli was marked on the hand of each subject who participated in the training phase. Care was taken to ensure that the position was constant across training sessions. A group of control subjects participated only in the pre- and post-test phases and did not undergo any training in the 2-3 weeks between the pre- and post-tests.

In addition to the somatosensory conditions, pre- and post-tests were conducted on auditory interval discrimination tests on one group. These tests were performed by procedures described by Wright et al. (1997a). In these tests the task was the same as in the somatosensory system, but stimulus markers were acoustic pulses (1 kHz tone pips, $15 \mathrm{msec}$ long, including $5 \mathrm{msec}$ rise/fall ramps, at an $86 \mathrm{~dB}$ sound pressure level) presented monaurally to the left ear through Sennheiser HD265 headphones. The tone pips were generated digitally with a Tucker-Davis Technologies (TDT) board and sampled at $25 \mathrm{kHz}$. Stimuli were matched to those used in the training experiments of Wright et al. (1997a), with base intervals of 50, 100, and $200 \mathrm{msec}$. Before and after training on 
A

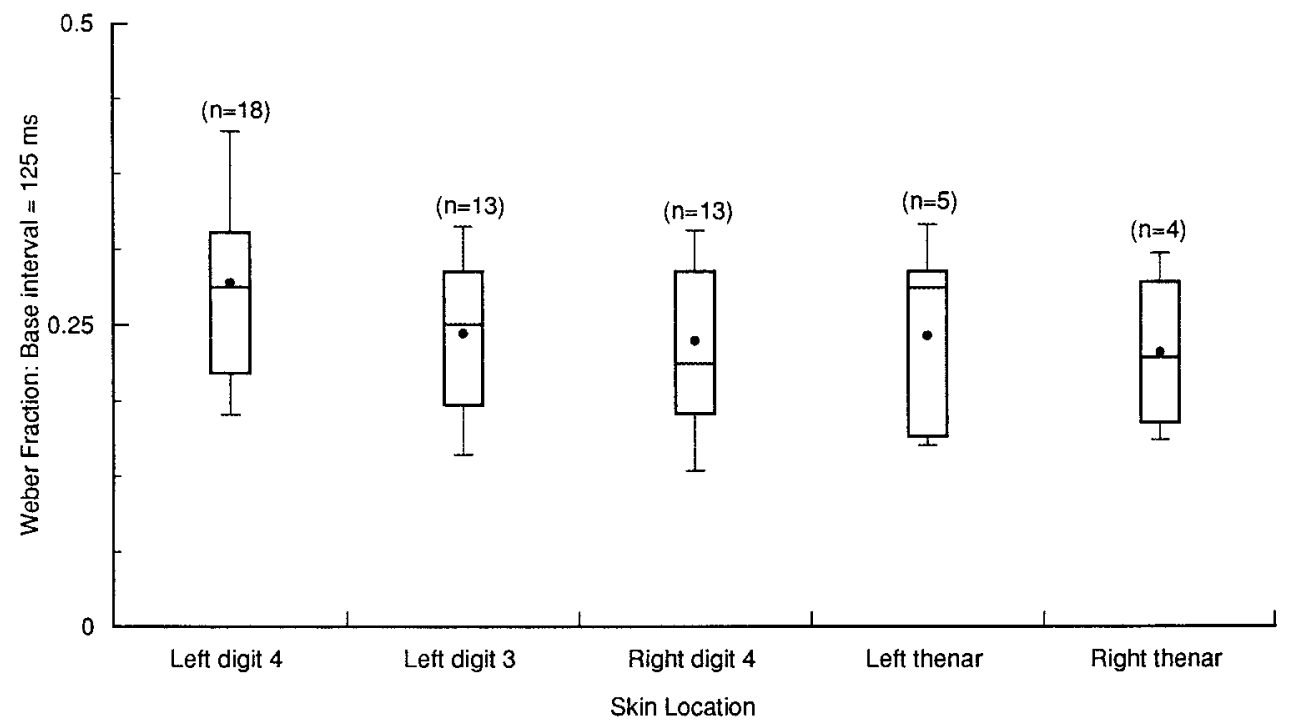

B

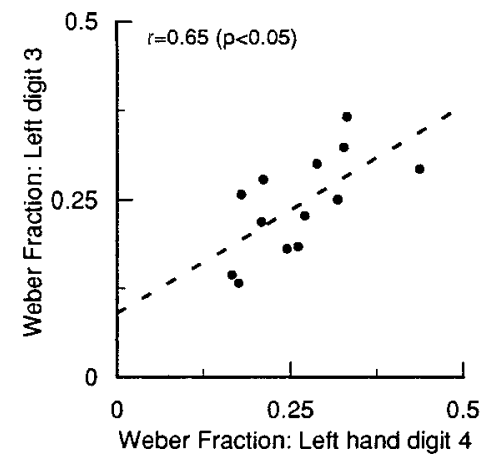

\section{C}

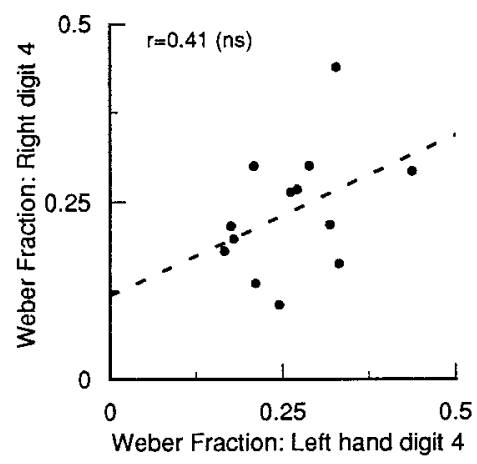

Figure 1. Effects of skin location. A, Distribution of interval discrimination thresholds expressed as Weber fractions for a base interval of $125 \mathrm{msec}$. Stimuli were delivered to five skin locations, as shown in the abscissa. The numbers in parentheses represent the number of subjects tested at each skin location. $B$, Interval discrimination thresholds for stimuli delivered to digit 4 of the left hand are plotted against thresholds for stimuli delivered to digit 3 of left hand. The linear regression fit is shown in the dashed line $(r=65 ; p<0.05)$. $C$, Interval discrimination thresholds for stimuli delivered to digit 4 of the left hand are plotted against thresholds for stimuli delivered to digit 4 of the right hand. The linear regression fit is shown in the dashed line ( $r=41 ; n s$, not statistically significant). interval discrimination in somesthesis, five or six blocks of 60 trials were collected for each of the three auditory base intervals. The auditory pre-and post-test measurements followed the somatosensory testing. The presentation order of the conditions was pseudo-randomized across subjects.

Changes in thresholds from the pre- to the post-test measurements determined the magnitude of learning in the trained condition and the magnitude of generalization attributable to learning in untrained conditions. To compare changes in thresholds across subjects with a wide range of thresholds, we found that it was necessary to normalize these changes to each subject's initial thresholds. Therefore, the characteristics of learning and generalization were evaluated from the fractional change in thresholds between the pre- and post-test phases in each condition. Fractional change was defined as:

Fractional change $=\frac{\text { Pretest Weber fraction }- \text { Post-test Weber fraction }}{\text { Pretest Weber fraction }}$.

Generalization to an untrained condition was considered complete if the mean fractional change in an untrained condition was greater than zero and not significantly different from the mean fractional change in the trained condition. Generalization was considered partial if fractional improvements in trained and untrained conditions were significantly different from zero and each other.

\section{RESULTS}

\section{Somatosensory temporal interval discrimination in naive subjects}

The first objective of this study was to determine the performance characteristics for somatosensory interval discrimination in naive subjects. This section describes three aspects of that performance.

\section{Skin location}

Thresholds for interval discrimination in somesthesis were similar across different skin locations in naive subjects. Figure $1 \mathrm{~A}$ shows pretest thresholds for interval discrimination, expressed as Weber fractions, for a base interval of $125 \mathrm{msec}$. Stimuli were delivered to the five skin locations listed along the abscissa. The number of subjects tested at each skin location is indicated in the parentheses. In this and subsequent figures, for each condition the dot represents the mean threshold, and the central line represents the median threshold. The box height shows the top and bottom quartile, and the outlier caps are placed on the top and bottom decile. A one-way factorial ANOVA with skin location as the factor indicated no significant differences for interval discrimination between spatial locations $\left(F_{(3,45)}=1.206 ; p>0.1\right)$. Interest- 


\section{A}

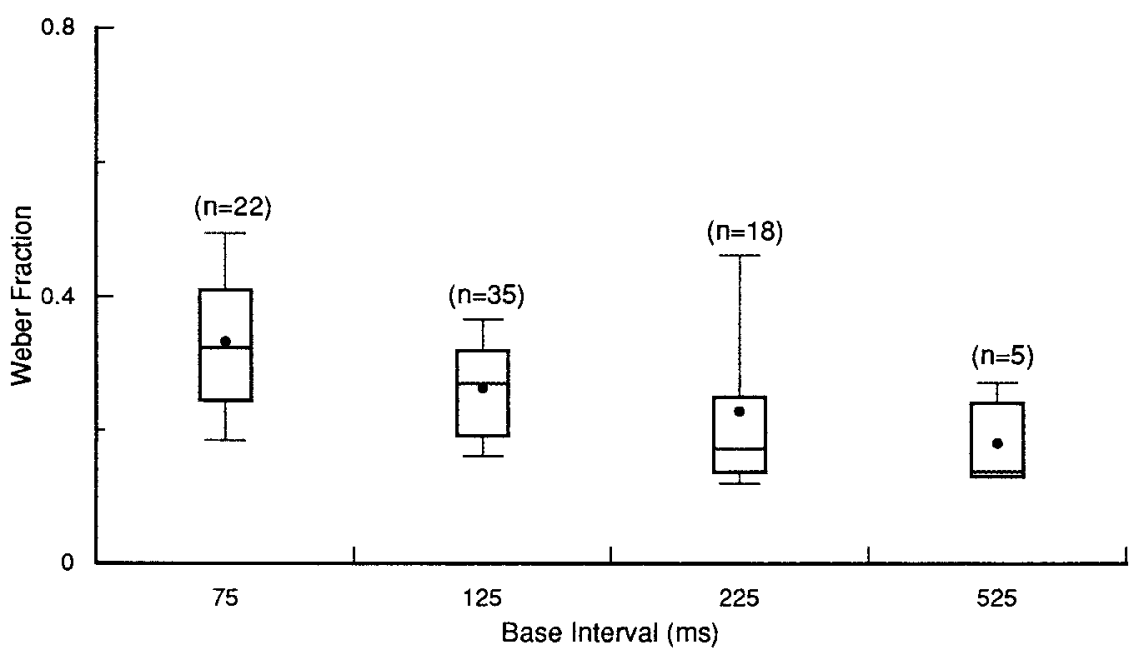

B

Figure 2. Effect of base interval and modality. $A$, Somatosensory interval discrimination thresholds for different base intervals expressed as Weber fractions. Thresholds at a base interval of $75 \mathrm{msec}$ are significantly different from thresholds at other base intervals. $B$, Distribution of interval discrimination thresholds plotted at different base intervals with somatosensory stimuli (base intervals of 75,125 , and 225 $\mathrm{msec}$ ) and auditory stimuli (base intervals of 50 , 100 , and $200 \mathrm{msec}$ ).

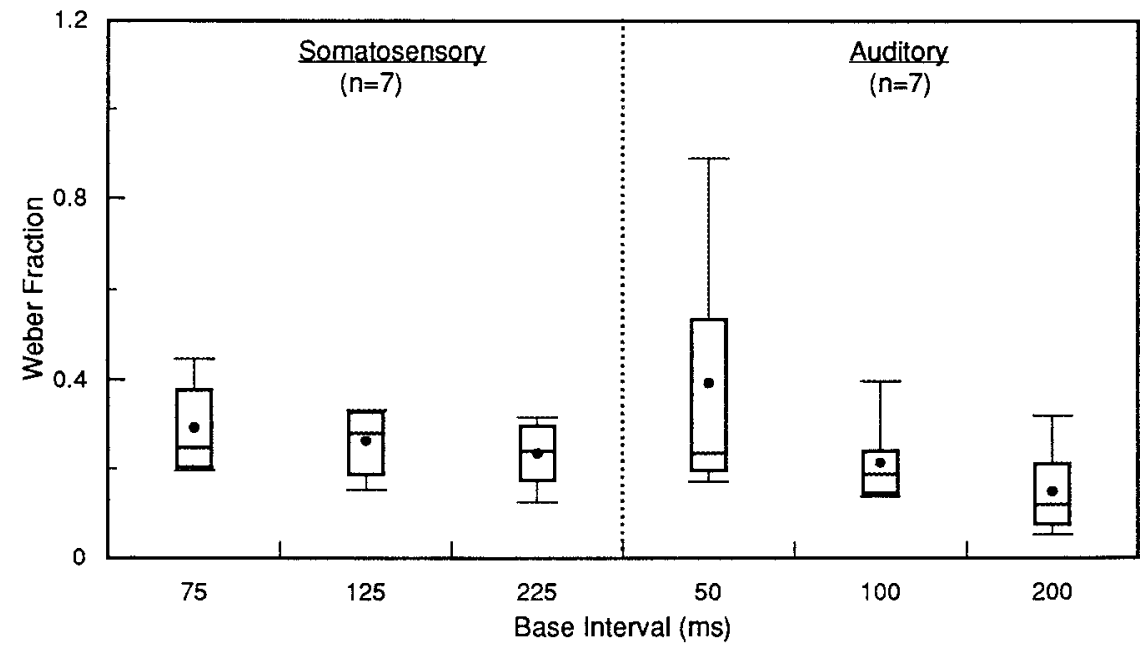

pooled across skin location. A one-way factorial ANOVA with base interval as the factor indicated significant differences in mean thresholds, expressed as Weber fractions, for different base intervals $\left(F_{(3,76)}=4.04 ; p<0.05\right)$. Post hoc Scheffé's and Bonferroni/Dunn tests indicated that only the thresholds for the base intervals of 75 and 225 msec were significantly different from each other $(p<0.005)$.

\section{Modality — somatosensory versus auditory}

Interval discrimination thresholds with somatosensory and auditory stimuli showed similar temporal precision across the two sensory systems for naive subjects. These data for both modalities obtained from the same set of seven subjects are shown in Figure $2 B$. A two-way factorial ANOVA with modality (auditory and somatosensory) and interval (50-75, 100-125, and 200-225 $\mathrm{msec})$ as factors revealed statistically significant threshold differences across intervals $\left(F_{(2,36)}=3.877 ; p<0.05\right)$, but not across modality $\left(F_{(1,36)}=0.06 ; p>0.5\right)$, with no significant interaction $\left(F_{(2,36)}=1.5 ; p>0.1\right)$. The thresholds for individual subjects for
$525 \mathrm{msec}$. Because there were no significant differences in thresholds for different skin locations, the data for this analysis were
Thresholds for somatosensory interval discrimination, expressed as Weber fractions, approached a constant value for longer base intervals and were significantly different for shorter base intervals, following a pattern similar to that for auditory duration discrimination (Getty, 1975). Figure $2 A$ shows Weber fraction thresholds for interval discrimination at base intervals of $75,125,225$, and 


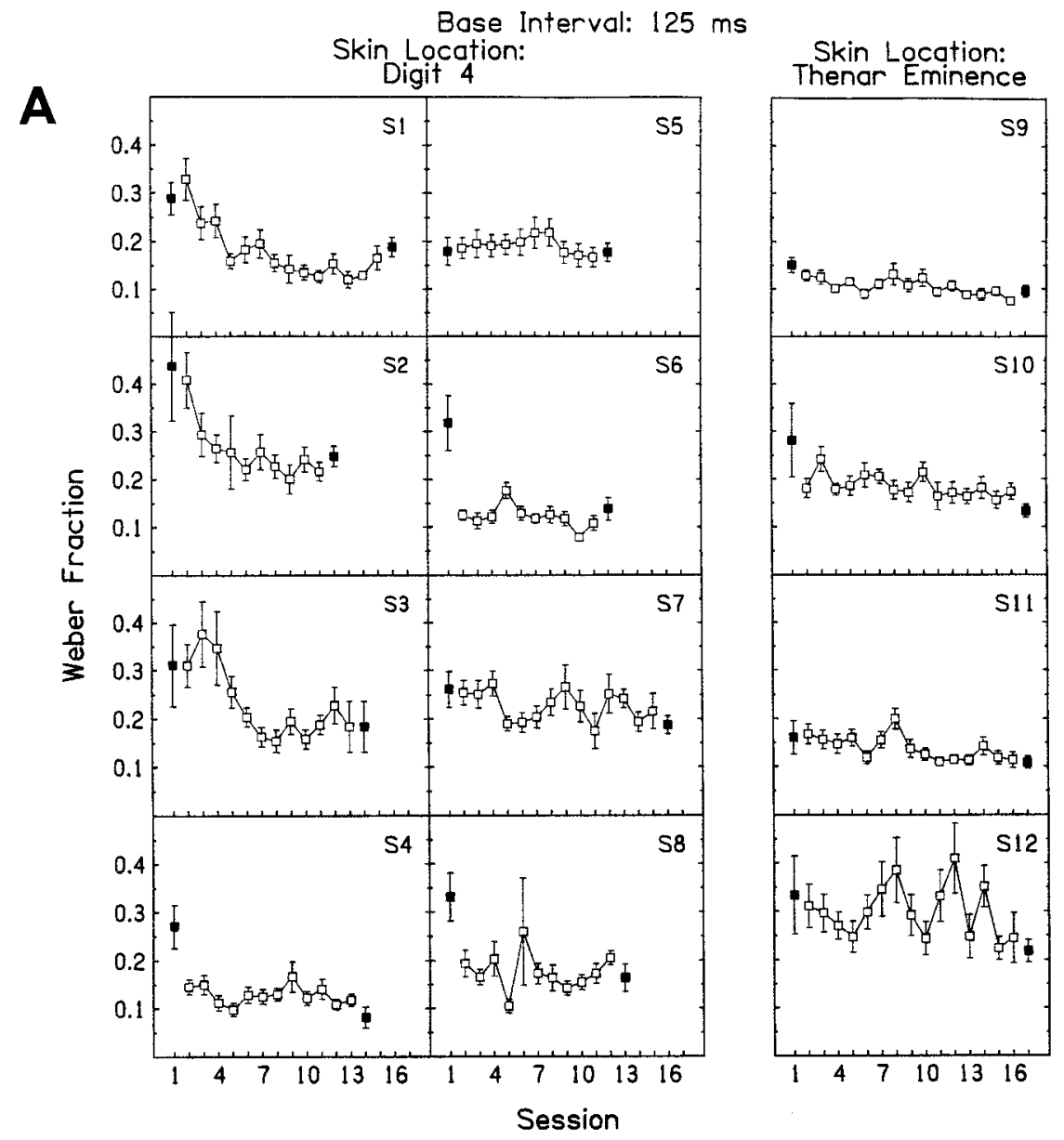

B

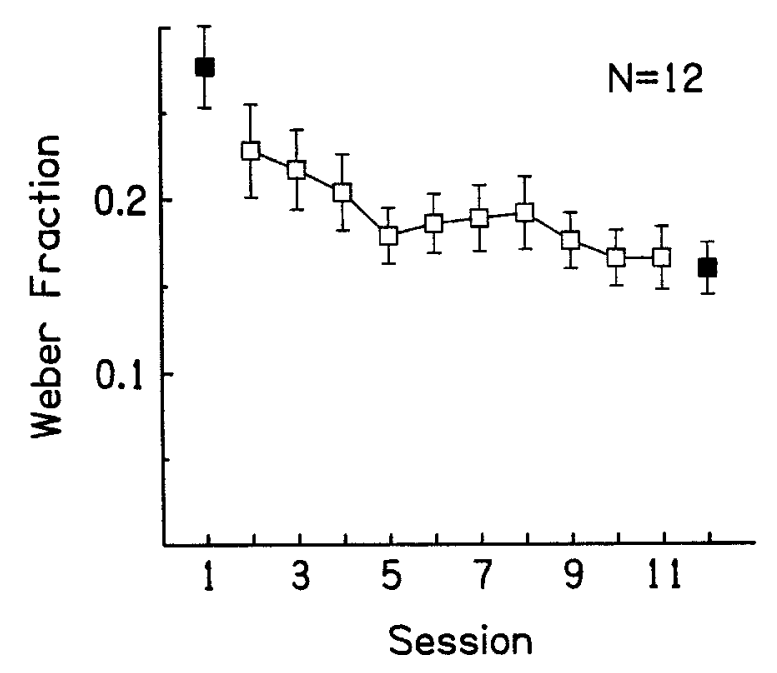

Figure 3. Learning curves for training at a base interval of $125 \mathrm{msec} . A$, Individual subject thresholds, expressed as Weber fractions, plotted as a function of training sessions. Stimuli were delivered to the distal tip of the fourth digit for subjects $S 1-S 8$ and to the thenar eminence for subjects $S 9-S 12$. Bars are SEM within subjects. The filled symbols indicate pre- and post-test thresholds for each subject. Note that the pre- and post-test thresholds were estimated from five to six blocks of 60 trials, whereas training thresholds were obtained for 15 blocks. $B$, Mean of all of the subjects trained at a base interval of $125 \mathrm{msec}$ plotted as a function of training days. Here, error bars represent 1 SEM across subjects. comparable base intervals were neither linearly nor rank orderly correlated between the two modalities, indicating idiosyncratic variability in thresholds across conditions.

\section{Perceptual learning of somatosensory interval discrimination}

The second objective of this study was to determine whether training improved somatosensory interval discrimination. Every subject improved with practice. The individual learning profiles for 12 subjects trained at a base interval of $125 \mathrm{msec}$ are shown in Figure $3 A$. The symbols indicate the mean thresholds obtained during each training session (open squares) and in the pre- and post-test phases (filled squares). The error bars indicate $\pm 1 \mathrm{SEM}$ within subjects. Note that each training session consisted of 10-15 threshold estimates, whereas the pre- and post-test values are each based on five to six threshold estimates. The eight panels on the left of Figure $3 A(S 1-S 8)$ show learning curves for subjects 
trained at the distal tip of the fourth digit of the left hand. The four panels on the right $(S 9-S 12)$ show learning for subjects trained on the thenar eminence of the left hand. Reductions in threshold, which indicated improvement, were observed in all of the subjects trained in the study. Some subjects made dramatic improvements (S1-S3), whereas others showed lesser improvements.

Figure $3 B$ shows the mean learning curves for the first $10 \mathrm{~d}$ of training for the 12 subjects who worked at the base interval of 125 msec. Performance improved rapidly in the first few days of training and then saturated at approximately the 10th day. Mean thresholds between the pretest and post-test phases changed by $42 \%$ (from a Weber fraction of $0.28-0.16$ ). A one-way repeated measures ANOVA with threshold over all of the days as the repeated measure revealed that this change was significant $\left(F_{(11,11)}=7.732 ; p<0.0001\right)$. When the pre- and post-test measurements were excluded from the analysis, the magnitude of the improvement was $30 \%$ (from a mean Weber fraction of $0.23-0.16)$ and was still significant $\left(F_{(11,9)}=3.3 ; p<0.005\right)$.

The observed improvements were not specific to training at 125 msec. Figure $4 A$ shows the individual learning curves, and Figure $4 B$ shows the mean learning curve for four subjects trained at a base interval of $75 \mathrm{msec}$. Mean performance improved significantly by $63 \%$ from an initial Weber fraction of 0.49 in the pretest to 0.18 in the post-test $\left(F_{(3,11)}=8.56 ; p<0.0001\right)$. Again, excluding the pre- and post-test measurements reduced the magnitude of improvement to $42 \%$ (from 0.33 to 0.19 ), which was still significant $\left(F_{(3,9)}=4.1 ; p<0.001\right)$.

To confirm that the observed changes were attributable to training, we measured threshold changes for a base interval of 125 msec in the untrained control group across pre- and post-test sessions separated by $10-14 \mathrm{~d}$ (see Fig. $5 A$ ). Data from the two trained groups and the control group were analyzed by a one-way factorial ANOVA for fractional change, with group as the factor. This analysis revealed a significant effect for group $\left(F_{(3,19)}=6.53\right.$; $p<0.005)$, and post hoc Scheffé's and Bonferroni/Dunn tests revealed that there were significant differences between the control group and both training groups $(p<0.01)$. Furthermore, changes in the control group were not significantly different from zero, as revealed by a Student's $t$ test on the fractional improvements for subjects in the control group $\left(t_{(5)}=0.853 ; p>0.1\right)$.

Finally, to address whether changes attributable to training correlated with pre- or post-test thresholds, fractional changes for individual subjects were plotted against their pretest and post-test thresholds (Fig. 5B). Fractional changes in the trained condition showed a moderate and statistically significant positive correlation with the pretest thresholds $(r=0.56 ; p<0.05)$, but not with the post-test thresholds (right panel). Consistent with this observation, the variability of the thresholds decreased both within and across subjects after training (Fig. 5C).

\section{Generalization of Learning in somatosensory interval discrimination}

The third objective of this study was to determine the generalization of learning in somatosensory interval discrimination. Three forms of generalization are described in this section.

\section{Spatial generalization}

Learning at the trained location generalized to untrained skin locations. Figure $6 \mathrm{~A}$ shows fractional changes for the $125 \mathrm{msec}$ base interval at the trained and untrained skin locations for all of the subjects trained at a base interval of $125 \mathrm{msec}$. Subjects
A

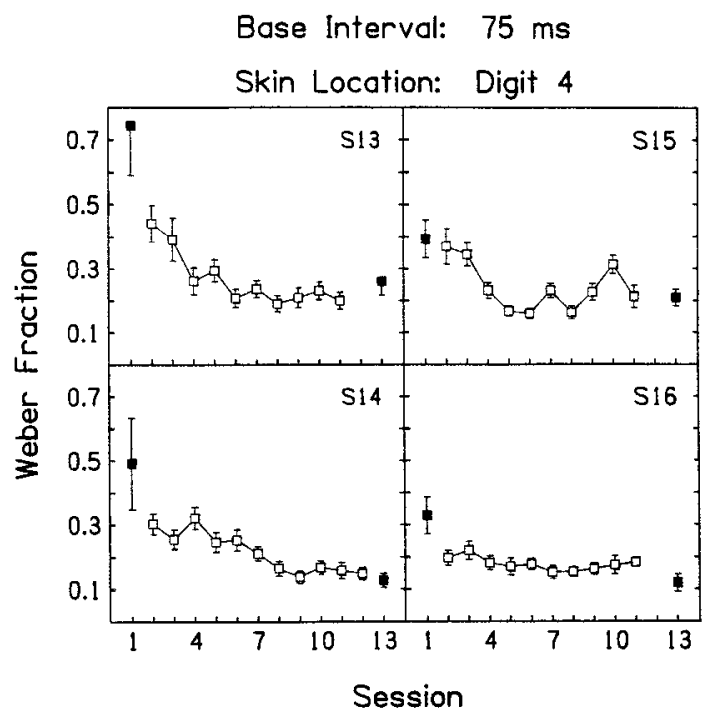

B

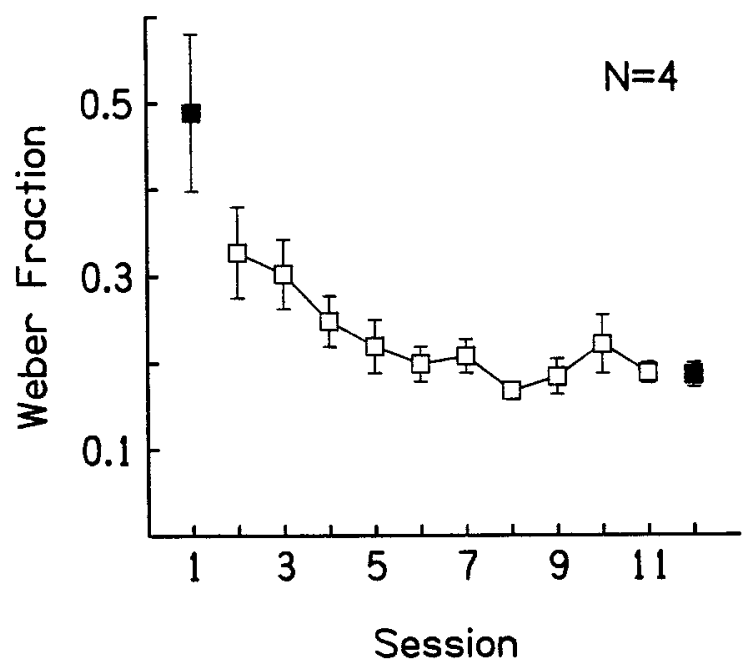

Figure 4. Learning curves for training at a base interval of 75 msec. $A$, Individual subject thresholds, expressed as Weber fractions, plotted as a function of training sessions. Stimuli were delivered to the distal tip of the fourth digit for subjects S13-S16. Bars are SEM within subjects. The filled symbols indicate pre- and post-test thresholds for each subject. Note that the pre- and post-test thresholds were estimated from five to six blocks of 60 trials, whereas training thresholds were obtained for 15 blocks. $B$, Mean of all four subjects plotted as a function of training days. Here, error bars represent 1 SEM across subjects.

were trained either at the tip of digit 4 of the left hand or to the thenar eminences. Learning in both groups of subjects generalized to untrained contralateral (corresponding positions on the right hand, $\left.t_{(10)} ; p<0.05\right)$ skin locations. In subjects trained at the tip of digit 4 of the left hand, learning generalized to the adjacent digit (digit 3 of the left hand, $t_{(6)}=4.59$; $p<0.005)$. These generalizations to adjacent and contralateral skin locations were found to be complete. A one-way ANOVA with trained and untrained skin location as the factor revealed no statistically significant differences among the fractional changes between trained and untrained skin locations $\left(F_{(2,26)}\right.$ $=1.82 ; p>0.1$ ). 
A

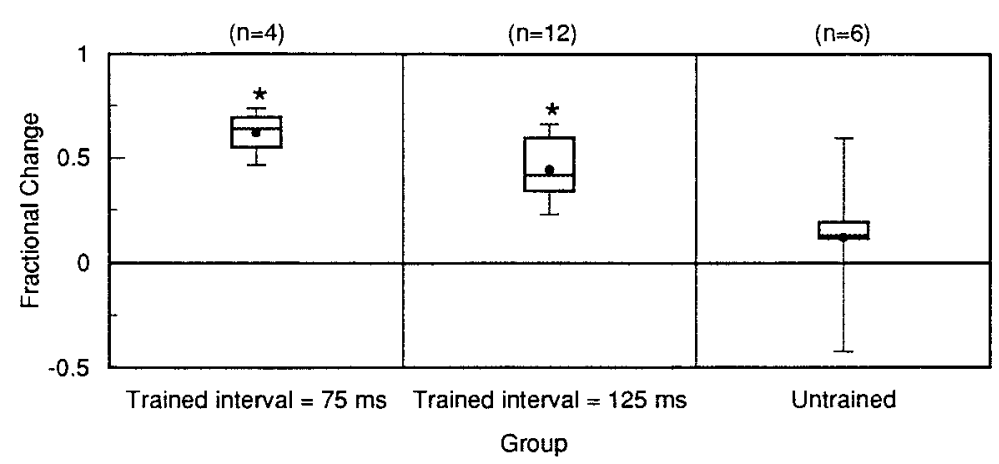

B
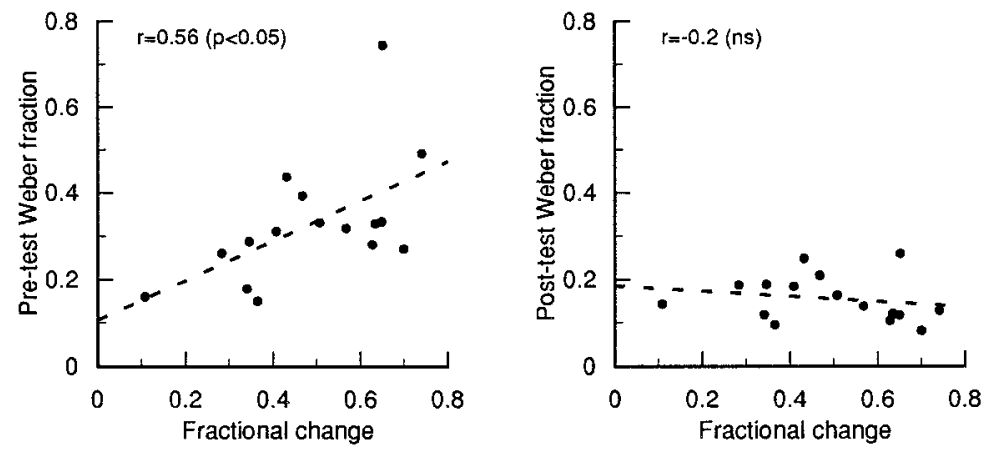

C
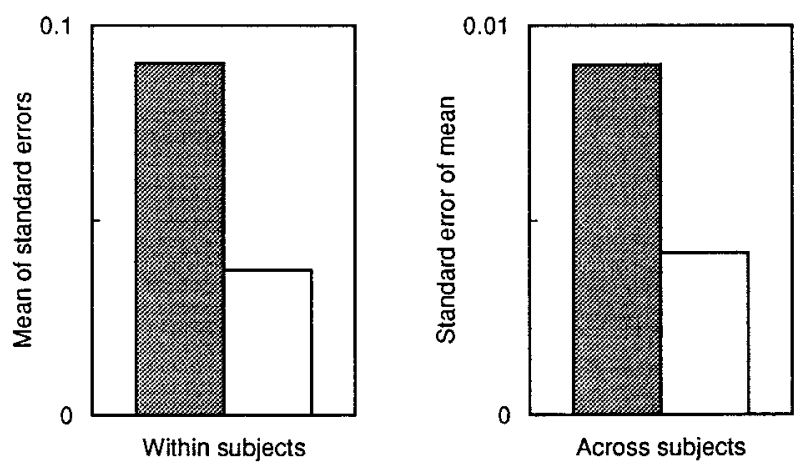

Pre-test

Post-test
Figure 5. Summary of learning. A, Distribution of fractional changes between pre- and post-tests are shown for three groups: (1) subjects trained at a base interval of $75 \mathrm{msec}$, (2) subjects trained at a base interval of $125 \mathrm{msec}$, and (3) untrained subjects tested with no training during the $10-15 \mathrm{~d}$ between pre- and post-tests. $B$, Individual subjects' pretest (left panel) and post-test thresholds (right panel) are plotted as a function of their fractional change in the trained condition. Dashed lines are linear regression fits, which account for $31 \%$ of the variance between pretest threshold and fractional changes $(r=0.56 ; p<0.05)$ and for $<4 \%$ of the variance between post-test thresholds and fractional changes $(r=-0.2, \mathrm{~ns}) . C$, The variability of thresholds decreases with training both within (left panel) and across subjects (right panel). Within subjects, the SEMs of average pre- and post-test thresholds are shown. Across subjects, the SEMs of the pre-and post-test thresholds are shown.

\section{Temporal generalization}

Learning at the trained base interval generalized only partially or not at all to untrained base intervals. To determine the temporal characteristics of generalization, we first examined fractional changes across subjects trained at a base interval of $125 \mathrm{msec}$, who were tested at untrained base intervals of 75 and $225 \mathrm{msec}$. For these analyses the fractional changes were pooled across subjects trained at different skin locations for the same base intervals. Figure $7 A$ shows that training at a base interval of $125 \mathrm{msec}$ completely generalized to the untrained interval of $75 \mathrm{msec}$ but showed no generalization to $225 \mathrm{msec}$. Mean fractional changes showed significant differences from zero at a base interval of 75 msec $\left(t_{(11)}=4 ; p<0.005\right)$, but not at a base interval of $225 \mathrm{msec}$ $\left(t_{(11)}=0.08 ; p>0.9\right)$. Further, a one-way factorial ANOVA performed with base intervals $(75,125$, and $225 \mathrm{msec})$ as the factors showed that mean fractional changes differed across base intervals $\left(F_{(3,37)}=3 ; p<0.05\right)$. Post hoc Scheffé's and Bonferroni/Dunn tests revealed that fractional changes for the base

interval of $225 \mathrm{msec}$ differed significantly from those for shorter base intervals $(p<0.01)$. Surprisingly, however, across subjects the fractional changes at base intervals of $75 \mathrm{msec}$ did not show any statistically significant linear or rank order correlation with fractional changes at a base interval of $125 \mathrm{msec}$.

Figure $7 B$ shows that training at a base interval of $75 \mathrm{msec}$ only partially generalized to an untrained interval of $125 \mathrm{msec}$, the only untrained interval tested. Mean fractional changes were significantly different from zero at the untrained base intervals of $125 \mathrm{msec}$, as revealed by a Student's $t$ test for fractional changes $\left(t_{(3)}=8.5 ; p<0.001\right)$. A one-way ANOVA performed with base interval (75 and $125 \mathrm{msec}$ ) as the factor showed that mean fractional changes differed across these base intervals $\left(F_{(1,6)}=\right.$ 8.03; $p<0.05)$.

\section{Modality generalization}

Interval discrimination learning in the somatosensory system generalized to the auditory system, but only for an auditory base 
Figure 6. Spatial generalization. Distribution of fractional changes at a base interval of $125 \mathrm{msec}$ for stimuli delivered to the trained skin location and an untrained skin location on the same hand and to an untrained but corresponding location on the contralateral hand is shown.

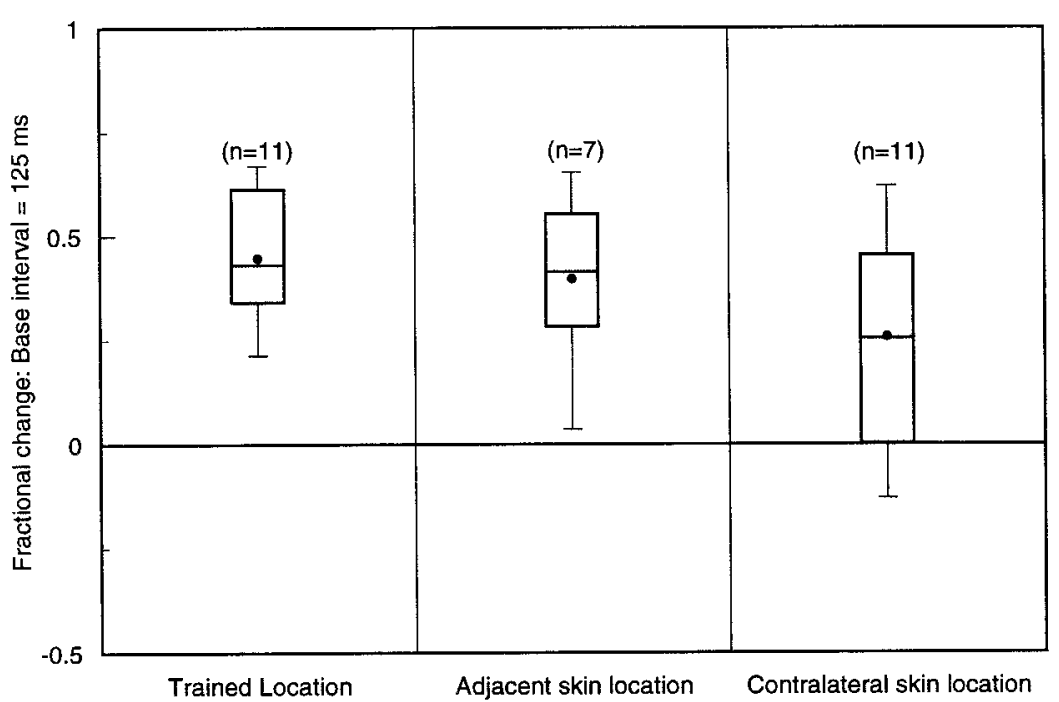

interval similar to the trained somatosensory one. To assess whether improvements in somatosensory interval discrimination transferred across modality to the auditory system, we determined fractional changes for the seven subjects tested on auditory interval discrimination before and after training in the somatosensory system at a base interval of $125 \mathrm{msec}$. Mean fractional changes with auditory stimuli were significantly different from zero only at a base interval of $100 \mathrm{msec}\left(t_{(6)}=16.4 ; p<0.0001\right.$; see Figure $8 A$ ). A two-way factorial ANOVA with modality (auditory and somatosensory) and intervals (50-75, 100-125, and 200-225 msec) as factors showed a significant main effect for base interval $\left(F_{(2,36)}=9.7 ; p<0.001\right)$, but not for modality. Post hoc Bonferroni/Dunn tests revealed that fractional changes were significantly different from each other only for a base interval of 200 msec with auditory stimuli $(p<0.01)$ and for a base interval of $225 \mathrm{msec}$ with somatosensory stimuli $(p<0.01)$. This suggests that interval discrimination learning in the somatosensory system generalized to the auditory system. However, generalization was complete only to an auditory base interval similar to the trained somatosensory base interval. Furthermore, partial-to-no generalization to the auditory system was observed at base intervals significantly different from the trained interval. Thus, learning in interval discrimination was specific to the trained base interval, but it was generalized for that interval across skin location and even modality. Consistent with this observation, there was a significant linear correlation between the fractional changes of individual subjects at the trained somatosensory base interval of $125 \mathrm{msec}$ and the untrained auditory base interval of $100 \mathrm{msec}$ (see Fig. $8 B ; r=0.89 ; p<0.05$ ).

\section{DISCUSSION}

\section{Performance of somatosensory interval discrimination in naive subjects}

The purpose of this study was to characterize somatosensory interval discrimination by naive subjects and to assess the capacity for perceptual learning and generalization of somesthetic interval discrimination. An important finding of this study was that interval discrimination by naive subjects within the somatosensory system is approximately equivalent to that reported for the auditory system (Wright et al., 1997a) and similar across base intervals, spatial location, and modality. These results suggest that discrimination of timing information across these two sensory systems may operate via similar central timing mechanisms.

The present data revealed few significant linear or rank order correlations between thresholds across different measurement conditions. This observation could result simply from the variability of thresholds in naive subjects. Alternatively, significant correlations or the lack thereof could reflect the mechanisms responsible for task performance. If the latter were true, the observation of a significant correlation between interval discrimination thresholds on adjacent digits would suggest that similar local timing machinery is involved in those discriminations. All of the subjects were right-handed, and no significant differences were observed between thresholds in the right hand and in the left. This lack of correlation of thresholds between contralateral skin locations and of thresholds with handedness would suggest an independence between temporal processing and handedness, consistent with previous reports on haptic processing and handedness (Summers and Lederman, 1990). Furthermore, the data do not reveal any significant correlations between naive thresholds with auditory and somatosensory stimuli. These data would suggest that, although central machinery-representing intervals are similar, they operate independently across modality and spatially distinct skin locations.

\section{Nontemporal cues and somatosensory interval discrimination}

Previous auditory studies have shown that nontemporal cues (e.g., perceptual effects on intensity, frequency, and bandwidth) are not used for duration discrimination with suprathreshold stimuli (Divenyi and Danner, 1977). Stimulus interactions, such as masking, suppression, or enhancement, do generate perceptual effects with somatosensory stimuli (Verillo and Gescheider, 1975; Gescheider and Migel, 1995). Several studies of Gescheider and colleagues have demonstrated masking interactions on detection thresholds up to $12 \mathrm{~dB}$ re $1 \mu \mathrm{m}$, which last up to $125 \mathrm{msec}$. However, clearly suprathreshold displacements were used in this study, so masking effects probably did not play a role in processing. With comparable stimuli, Verrillo et al. (1975) have shown that the perceived intensity of a stimulus pair (summation effect) or the subjective magnitude of the second stimulus of a pair (enhancement effect) were $<3 \mathrm{~dB}$ re $1 \mu \mathrm{m}$ displacements, as compared with the perceived intensity of a stimulus in isolation. Moreover, and 
A

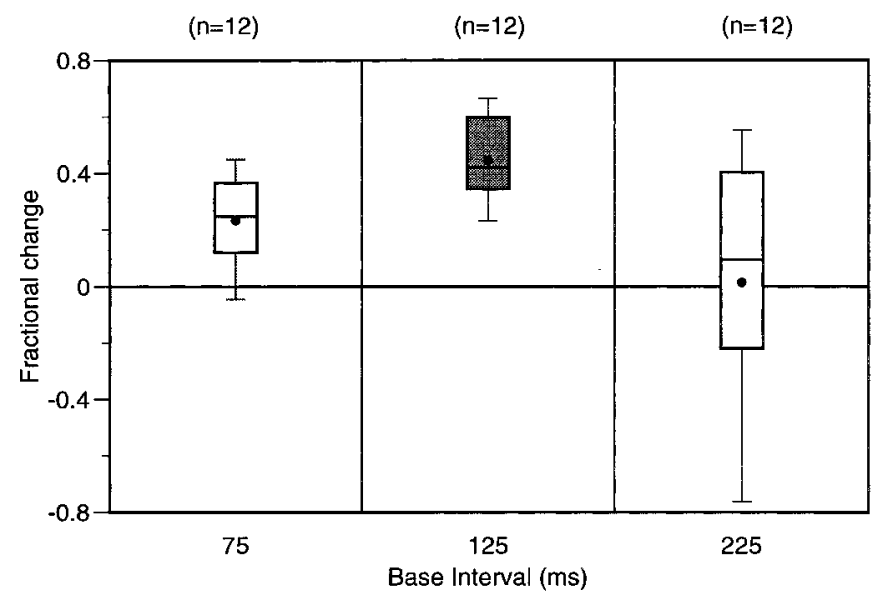

B

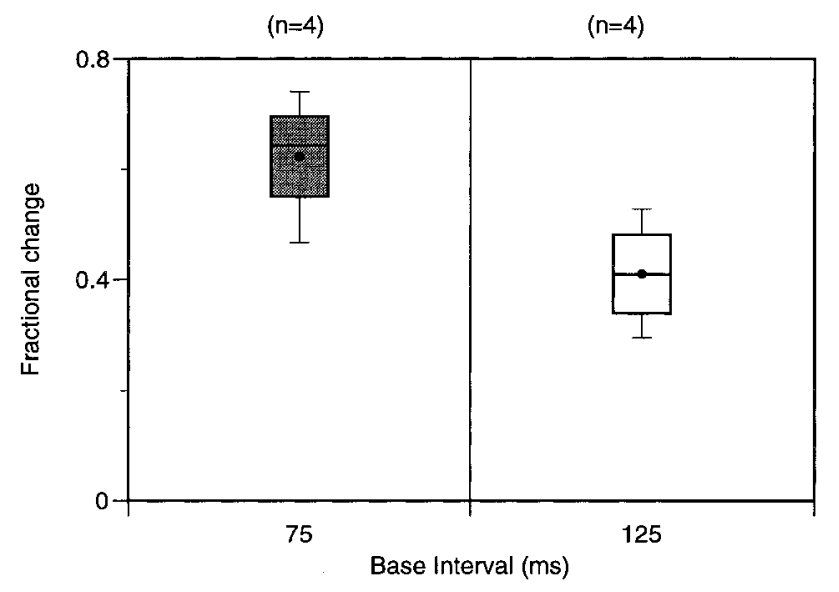

Figure 7. Temporal generalization. $A$, Distribution of fractional change at base intervals of 75,125 , and $225 \mathrm{msec}$ for subjects trained at a base interval of $125 \mathrm{msec}$ (indicated by the gray box). B, Distribution of fractional change at base intervals of 75 and $125 \mathrm{msec}$ for subjects trained at a base interval of $75 \mathrm{msec}$ (indicated by the gray box).

perhaps most significantly, summation and enhancement effects varied $<1 \mathrm{~dB}$ in the time scale of $75-525 \mathrm{msec}$. Therefore, it is unlikely that our subjects used nontemporal cues for performing interval discrimination.

\section{Perceptual learning of somatosensory interval discrimination}

The training data presented here represent the first demonstration of perceptual learning of interval discrimination in the somatosensory system. The time course of learning observed in this study is consistent with that observed for auditory interval discrimination (Michon, 1963; Aiken, 1965; Warm et al., 1975; Woods et al., 1979; Kristofferson, 1980; Wright et al., 1997a) and for visual spatial tasks such as orientation discrimination, vernier acuity, or pop-out detection (Ahissar and Hochstein, 1996).

Practice-related reductions in the variability of interval discrimination thresholds both within a given subject and across subjects indicate that training results in a convergence of performance to a similar asymptote. These performance limits may indicate the ultimate capacities and constraints for interval or duration estimation mechanisms in normal humans.

An earlier study that recorded no learning effects for interval discrimination concluded that a clock or timing mechanism that was affected by various internal or external factors would not be a very reliable clock (Rammsayer, 1994). In these studies the subjects were trained only on 50 trials per session. By contrast, the subjects in the present study were trained with 900 trials per session. Differences in training intensity, schedule, and trial numbers easily could account for differences in recorded improvements resulting from practice. The learning results reported here provide further evidence that, like many other cortical functions, timing mechanisms can be modified via training.

\section{Generalization of somatosensory interval discrimination}

Learning of somatosensory interval discrimination at the trained base interval generalizes completely to untrained skin locations. This finding is consistent with results obtained for interval discrimination that used auditory stimuli, in which training effects generalized across at least two octaves in the frequency dimension for the trained base interval (Wright et al., 1997a). However, it should be remembered that only nearby skin locations on either hand and corresponding skin locations across hands were examined in these experiments. The topographic specificity of interhemispheric cortical connections necessitates a cautionary interpretation of widespread spatial generalization. These results are also consistent with other studies on somatosensory perceptual learning that do not involve purely temporal tasks. For example, training on grating discrimination showed widespread spatial generalization but was highly specific to the trained task (Sathian and Zangaladze, 1997).

Somatosensory interval discrimination learning shows partial generalization to base intervals that are similar to the trained one and no generalization to farther base intervals. This temporal specificity is in contrast to auditory interval discrimination in which learning at a base interval of $100 \mathrm{msec}$ did not generalize to base intervals of 50 and $200 \mathrm{msec}$ (Wright et al., 1997a). The broader temporal generalization in the somatosensory system is consistent with the fact that other temporal interactions, such as masking, exhibit longer time constants in the somatosensory than in the auditory system (Gescheider and Migel, 1995). These results also support the notion that learning in temporal tasks is specific to the temporal information in stimuli, analogous to learning in spatial tasks, which is specific to the spatial aspects in stimuli (Karni and Sagi, 1991, 1993).

Remarkably, the observed temporal specificity for base interval extends to a second (auditory) modality. These results corroborate that the subjects in this study did not use nontemporal cues for task performance, because such a possibility could not account for the observed temporal specificity with auditory stimuli. The cross-modal generalization reported here is consistent with other reports on symmetric transfer of improvements in temporal discrimination accuracy between audition and vision attributable to training in audition (Warm et al., 1975; Rousseau et al., 1983). Such cross-modal generalization is also consistent with other perceptual learning experiments involving spatial discrimination tasks with vibrotactile patterns that transfer to vision (Epstein et al., 1989; Hughes et al., 1990). These previous and present examples of cross-modal generalization provide supportive evidence for "sensory integration training" (Ottenbacher, 1982).

It is also remarkable that there is a significant correlation 
A

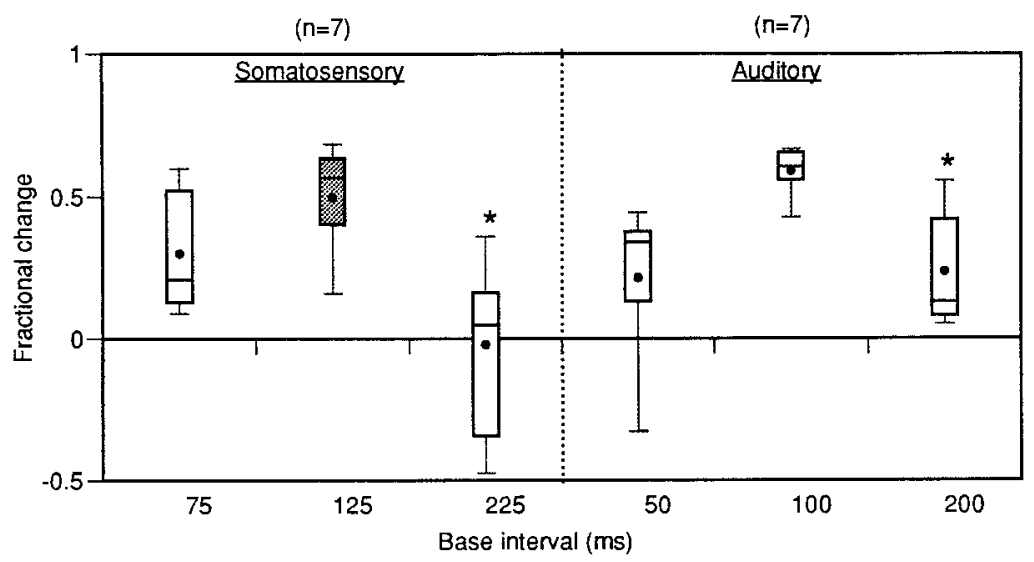

Figure 8. Generalization across modality: somatosensory to auditory. $A$, Distributions of fractional changes in interval discrimination for somatosensory stimuli (at base intervals of 75,125 , and $225 \mathrm{msec}$ ) and for untrained auditory stimuli (at base intervals of 50,100, and $200 \mathrm{msec}$ ). Note that the $125 \mathrm{msec}$ somatosensory base interval is the trained condition ( gray box). Fractional changes at $225 \mathrm{msec}$ with somatosensory stimuli and at $200 \mathrm{msec}$ with auditory stimuli are significantly different from the others (shown by asterisks). B, Fractional changes in the trained condition plotted against fractional changes in auditory interval discrimination at a base interval of $100 \mathrm{msec}$. The data show a moderate linear correlation of 0.89 ; a linear regression fit (dashed line) accounts for $78 \%$ of the variance.

B

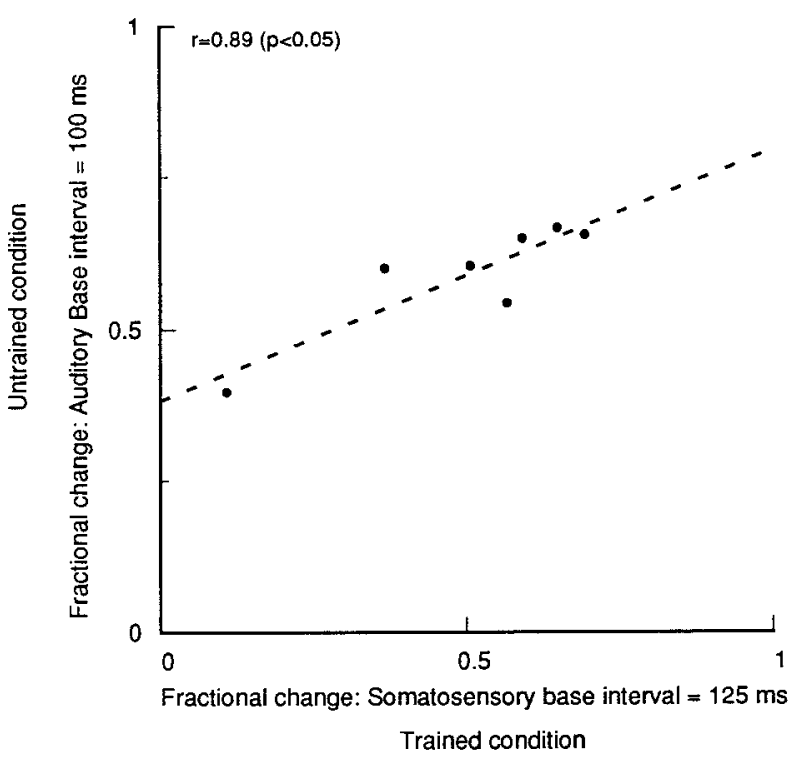

between improvements on a trained somatosensory condition and an untrained auditory condition at a comparable base interval (see Fig. 8). This correlation between fractional changes is not the consequence of a correlation between performance in pre- or post-test measurements, because there was no significant crossmodal correlation of performance on either of those conditions separately, and only the fractional change was correlated. Such generalization of the mean fractional changes across modality (to audition) also suggests that learning may occur via central or shared timing mechanisms, representing intervals that are independent of modality.

\section{Models for temporal processing}

Our findings, and those of a previous study (Wright et al., 1997a), provide evidence that challenges previous models of temporal processing. Both studies have found specificity in learning to the trained intervals, and both have found spatial transfer. Transfer to different frequencies in the auditory task is analogous to spatial

transfer in somesthesis. Whatever the mechanisms that underlie temporal processing in the CNS, they must be specific for interval length but less specific spatially.

Although several models have been proposed for perceptual learning and associated neural plasticity for spatial tasks, there are no widely accepted models for perceptual learning of temporal tasks because of the lack of information on the characteristics of learning associated with temporal information processing. Extensive studies on interval discrimination performance in experienced subjects have contributed to the formulation of several models of performance on temporal tasks.

One dominant model for temporal processing that is consistent with numerous psychophysical studies is the "internal clock" hypothesis. According to this hypothesis, a pacemaker generates pulses, and the number of pulses occurring during a given time period is recorded by an accumulator. The neurobiological bases for these pacemakers and accumulators currently are unknown, although cerebral oscillations have been argued to participate in 
such processing (Treisman, 1984, 1990, 1994). Internal clock models predict that the clock or timing mechanisms would be unaffected by external influences. Such models require significant modification if they are to account for the observed learning in interval discrimination.

An alternative model for interval discrimination is the interval measure hypothesis. Ivry and others have postulated a spatial representation of interval information in the brain that could be used in timing tasks (Ivry, 1996). They argue that increased Weber fractions for stimuli delivered across modalities could not be accounted for by internal clock models. Biologically plausible implementations of interval measure models usually assume the presence of a temporal-to-spatial transformation resulting from deterministic or stochastic "delay lines." Deterministic delay line models assume a range of time constants that represent intervals. Such models have been successful to a certain extent in implementing temporal tasks (Tank and Hopfield, 1987; Grossberg and Schmajuk, 1989). Stochastic delay line models do not assume a range of time constants. Rather, they postulate that timedependent neural and synaptic properties, such as short-term synaptic plasticity and slow inhibitory processes, participate in the emergent representation of intervals in the time scale of 50-500 msec (Buonomano and Merzenich, 1995). Recently, experimental evidence for the role of time-dependent neural properties on the representation of interval and duration has been reported (Casseday et al., 1994; Buonomano et al., 1997). Preliminary attempts at determining synaptic learning rules for the processing of temporally complex inputs have brought forth the realization that simplistic Hebbian principles need to be redefined in the context of complex time structure in sensory input (Buonomano et al., 1997; Markram et al., 1997a).

Our results and those of Wright et al. (1997a) showing temporal specificity and spatial generalization indicate that both the internal clock models and interval measure models must be modified significantly, because these models in their current forms would predict neither interval discrimination learning nor the characteristics of generalization. Moreover, both the internal clock and interval measure models assume a representation of absolute time intervals in contrast to the characteristics of relative time discrimination reported in this paper. Therefore, biologically realistic models for interval discrimination require the integration of the characteristics of learning reported here and a greater understanding of the neurological and psychophysical rules governing plasticity in the neural representation of temporal information.

\section{REFERENCES}

Ahissar M, Hochstein S (1996) Perceptual learning. In: Perceptual constances: why things look as they do (Walsh V, Kulikowsky J, eds). New York: Cambridge UP.

Aiken LR (1965) Learning and retention in the estimation of short time intervals: a circuit and a study. Percept Mot Skills 20:509-517.

Allan LG (1979) The perception of time. Percept Psychophys 26:340-354.

Allan LG (1992) The internal clock revisited. In: Time, action, and cognition: towards bridging the gap (Macar F, Pouthes V, Friedman WT, eds), pp 191-202. NATO ASI Series. Boston: Kluwer.

Buonomano DV, Merzenich MM (1995) Temporal information transformed into a spatial code by a neural network with realistic properties. Science 267:1028-1030.

Buonomano DV, Hickmott PW, Merzenich MM (1997) Contextsensitive synaptic plasticity and temporal-to-spatial transformations in hippocampal slices. Proc Natl Acad Sci USA 94:10403-10408.

Casseday JH, Ehrlich D, Covey E (1994) Neural tuning for sound dura- tion: role of inhibitory mechanisms in the inferior colliculus. Science 264:847-850.

Divenyi PL, Danner WF (1977) Discrimination of time intervals marked by brief acoustic pulses of various intensities and spectra. Percept Psychophys 21:125-142.

Drullman R (1995) Temporal envelope and fine structure cues for speech intelligibility. J Acoust Soc Am 97:585-592.

Epstein W, Hughes B, Schneider SL, Bach-y-Rita P (1989) Perceptual learning of spatiotemporal events: evidence from an unfamiliar modality. J Exp Psychol Hum Percept Perform 15:28-44.

Gescheider GA, Migel N (1995) Some temporal parameters in vibrotactile forward masking. J Acoust Soc Am 98:3195-3199.

Getty DJ (1975) Discrimination of short temporal intervals: a comparison of two models. Percept Psychophys 18:1-8.

Gibbon J, Church RM (1990) Representation of time. Cognition 37:23-54.

Gibbon J, Malapani C, Dale CL, Gallistel CR (1997) Toward a neurobiology of temporal cognition: advances and challenges. Curr Opin Neurobiol 7:170-184.

Grossberg S, Schmajuk NA (1989) Neural dynamics of adaptive timing and temporal discrimination during associative learning. Neural Networks 338:334-337.

Hughes B, Epstein W, Schneider S, Dudock A (1990) An asymmetry in transmodal perceptual learning. Percept Psychophys 48:143-150.

Ivry R (1996) The representation of temporal information in perception and motor control. Curr Opin Neurobiol 6:851-857.

Karni A, Sagi D (1991) When practice makes perfect in texture discrimination: evidence for primary visual cortex plasticity. Proc Natl Acad Sci USA 88:4966-4970.

Karni A, Sagi D (1993) The time course of learning a visual skill. Nature $365: 250-252$.

Koczmarec KA, Webster JG, Tompkins WM (1991) Electrotactile and vibrotactile displays for sensory substitution systems. IEEE Trans Biomed Eng 38:1-16.

Kristofferson AB (1980) A quantal step function in duration discrimination. Percept Psychophys 27:300-306.

Levitt H (1971) Transformed up-down methods in psychoacoustics. J Acoust Soc Am 60:1199-1202.

Licklider JCR (1951) The perception of speech. In: Handbook of experimental psychology (Stevens SS, ed). New York: Wiley.

Markram H, Lubke J, Frotscher M, Sakmann B (1997) Regulation of synaptic efficacy by coincidence of postsynaptic APs and EPSPs. Science 275:213-215.

Merzenich MM, Jenkins WM, Johnston P, Schreiner C, Miller SL, Tallal P (1996) Temporal processing deficits of language-learning impaired children ameliorated by training. Science $271: 77-81$.

Michon JA (1963) Studies on subjective duration. Acta Psychol (Amst) 22:441-450.

Minagawa H, Ohnishi N, Sugie N (1996) Tactile-audio diagram for blind persons. IEEE Trans Rehabil Eng 4:431-437.

Mountcastle VB (1993) Temporal order determinants in a somesthetic frequency discrimination: sequential order coding. Ann NY Acad Sci 682:150-170.

Ottenbacher K (1982) Sensory integration therapy: affect or effect. Am J Occup Ther 36:571-578.

Rammsayer TH (1994) Effects of practice and signal energy on duration discrimination of brief auditory intervals. Percept Psychophys 55:454-464.

Rousseau R, Poirier J, Lemyre L (1983) Duration discrimination of empty time intervals marked by intermodal pulses. Percept Psychophys 34:541-548.

Sathian K, Zangaladze A (1997) Tactile learning is task specific but transfers between fingers. Percept Psychophys 59:119-128.

Shannon RV, Zeng FG, Kamath V, Wygonski J, Ekelid M (1995) Speech recognition with primarily temporal cues. Science 270:303-304.

Summers DC, Lederman SJ (1990) Perceptual asymmetries in the somatosensory system: a dichhaptic experiment and critical review of the literature from 1929 to 1986 . Cortex 26:201-226.

Tallal P (1990) Fine-grained discrimination deficits in language-learning impaired children are specific neither to the auditory modality nor to speech perception. J Speech Hear Res 33:616-619.

Tallal P, Miller S, Fitch RH (1993) Neurobiological basis of speech: a case for the preeminence of temporal processing. Ann NY Acad Sci 682:27-47. 
Tank DW, Hopfield JJ (1987) Neural computation by concentrating information in time. Proc Natl Acad Sci USA 84:1896-1900.

Treisman M (1984) Temporal rhythms and cerebral rhythms. Ann NY Acad Sci 423:542-565.

Treisman M, Faulkner A, Naish PLN, Brogan D (1990) The internal clock: evidence for a temporal oscillator underlying time perception with some estimates of its characteristic frequency. Perception 19:705-743.

Treisman M, Cook N, Naish PLN, MacCrone JK (1994) The internal clock: electroencephalographic evidence for oscillatory processes underlying time perception. Q J Exp Psychol [A] 47:241-289.

Verillo RT, Gescheider GA (1975) Enhancement and summation in the perception of two successive vibrotactile stimuli. Percept Psychophys 18:128-136.

Warm JS, Stutz RM, Vassolo PA (1975) Intermodal transfer in temporal discrimination. Percept Psychophys 18:281-286.

Woods DD, Sorkin RD, Boggs GJ (1979) Stimulus context and duration discrimination. Percept Psychophys 26:127-132.

Wright BA, Buonomano D, Mahncke H, Merzenich M (1997a) Learning and generalization of auditory temporal interval discrimination. J Neurosci 17:3956-3963.

Wright BA, Lombardino LJ, King WM, Puranik CS, Leonard CS, Merzenich MM (1997b) Deficits in auditory temporal and spectral resolution in language-impaired children. Nature 387:176-178. 